Archives

$6 \mid 1990$

Varia

\title{
Forêts d'Europe au Moyen-Âge
}

\section{Philippe Braunstein}

\section{OpenEdition \\ Journals}

Édition électronique

URL : http://journals.openedition.org/ccrh/2859

DOI : $10.4000 /$ ccrh.2859

ISSN : 1760-7906

Éditeur

Centre de recherches historiques - EHESS

Édition imprimée

Date de publication : 15 octobre 1990

ISSN : 0990-9141

Référence électronique

Philippe Braunstein, «Forêts d'Europe au Moyen-Âge », Les Cahiers du Centre de Recherches Historiques [En ligne], 6 | 1990, mis en ligne le 20 mars 2009, consulté le 19 avril 2019. URL : http:// journals.openedition.org/ccrh/2859; DOI : 10.4000/ccrh.2859

Ce document a été généré automatiquement le 19 avril 2019

Article L.111-1 du Code de la propriété intellectuelle. 


\title{
Forêts d'Europe au Moyen-Âge
}

\author{
Philippe Braunstein
}

1 L'histoire de la forêt à l'époque médiévale offre un exemple caractéristique de l'évolution du regard sur un espace, défini à la fois comme un territoire hostile et comme un lieu de vie et de production. La forêt est d'abord un monde de confins, barrière hostile ou protectrice, cantonnée par des aménagements défensifs que César a décrits en Belgique, et qui subsistent encore dans des paysages fossiles d'Europe centrale et orientale aux limites des communautés villageoises: les grandes haies forestières ne sont pas les clôtures arbustives, terme dernier du bois fermé face aux prés et aux champs, mais des rangées d'arbres imbriqués les uns dans les autres par des branches taillées et courbées à hauteur d'homme. Au delà de cette barrière, plus ou moins organisée, s'étend un monde considéré comme dangereux, refuge des marginaux et des hors-la-loi, désert spirituel des ascètes.

2 Réserve de vie sauvage, qui orne la table des grands et nourrit leur imaginaire, la forêt est par ailleurs, au moins dans ses marges, constamment associée à l'élevage; lieu de production du principal voire du seul combustible qu'est le charbon de bois, elle est indispensable à la pratique quotidienne de nombreux métiers ruraux et urbains.

3 Les deux définitions ne cessent de coexister, mais le regard évolue au fur et à mesure que s'affirme la maitrise de l'espace forestier par les populations qui l'habitent et l'exploitent. $\mathrm{Au} \mathrm{XI}^{\mathrm{e}}$ siècle, on parlait en Ile-de-France de l'Yveline, de la Bière, de la Laye pour définir des massifs forestiers compacts et par endroits presque impénétrables comme des entités vivantes; au XIII ${ }^{\mathrm{e}}$ siècle, les zones épargnées par les défrichements ont pris le nom de l'agglomération la plus proche, et l'on parle de la forêt de Rambouillet, de Fontainebleau ou de Saint-Germain. Ces zones stabilisées sont dès lors les éléments d'un patrimoine circonscrit, arpenté et, après le XVI siècle, cartographié. Depuis le XI siècle en Angleterre ${ }^{1}$, et à partir du XIII ${ }^{\mathrm{e}}$ siècle dans toute l'Europe, les sources écrites apportent la preuve que la forêt, assise de droits et de coutumes, est conçue comme une base appréciable de revenus justiciers et fonciers.

4 Le problème qui s'est posé aux propriétaires, aux seigneurs éminents et aux législateurs a été d'assurer la fructification des revenus sans en épuiser la source : tirer de la forêt tout 
ce qu'elle recèle de ressources, du miel à la mine de fer, sans que l'exploitation de ces ressources nuise à l'entretien et à la survie des massifs forestiers. C'est-à-dire que, depuis la loi des Burgondes, dans les traces écrites, se trouvent en présence des utilisateurs de la forêt et des droits qui en grèvent les revenus, dont les intérêts risquent de s'opposer, et que les pouvoirs tentent de faire coexister; c'est une situation que l'essor démographique, les besoins des consommateurs, rendent, avec le temps, plus aigüe. Réserve inépuisable et incommensurable au haut Moyen Age, où faute d'arpenter les bois, on les mesure en nombre de porcs à nourrir dans la forêt proche et en jours de marche de traversée dans la forêt lointaine, les massifs boisés sont à la mesure de la croissance, des innovations techniques et de la circulation accrue des biens de consommation, soumis à des ponctions telles que se développe, dès le XIII ${ }^{\mathrm{e}}$ siècle en certaines régions, le thème obsessionnel du manque de bois. Les controverses entre utilisateurs et parties prenantes - communautés villageoises, bourgeois et seigneurs industriels, autorités municipales l'argumentation hostile à l'industrie analysée pour l'époque moderne par Denis Woronoff ${ }^{2}$, se retrouvent dans des procès et des rapports au seigneur à partir du XIV ${ }^{\mathrm{e}}$ siècle, avec la tentation pour les pouvoirs publics de résoudre par la force - la destruction des forges prédatrices de la forêt - des problèmes d'adaptation de la demande à l'offre' .

Ces débats ont l'avantage de mettre en lumière les signes d'une volonté économique et politique de protection des ressources de la forêt: non le souci de conserver à l'environnement boisé ses vertus naturelles, mais - le bois étant le combustible à peu près unique des sociétés industrielles-le désir de favoriser la création ou la survie d'entreprises. Cette prise de conscience, avec ses aspects positifs et négatifs, l'inquiétude plus ou moins fondée devant le manque de bois, ou la détermination à gérer raisonnablement l'espace forestier ${ }^{4}$, est un phénomène majeur dont l'étude est à poursuivre.

6 On doit aux travaux de synthèse de Ch. Higounet une courageuse tentative de cartographier l'extension minimale des forêts d'Occident avant l'an mil ${ }^{5}$, sans que pour autant les limites et la compacité des massifs soient certaines, et sans que les dates des plus anciennes mentions connues apportent plus qu'un point de repère. C'est la couverture forestière de l'Angleterre qui est la mieux connue pour l'époque saxonne, grâce aux recherches des archéologues et des palinologues ${ }^{6}$. De façon générale, un contraste frappant s'établit dès le haut Moyen Age et se renforce par la suite entre une forêt méditerranéenne fragile et dont l'érosion paraît irréversible, et les ressources considérables de la forêt océane et germanique, qui ont alimenté le grand courant d'exportation de bois à l'époque carolingienne vers le monde arabe, décrit et cartographié par M. Lombard7 .

7 L'histoire de la forêt européenne est longtemps restée dans l'ombre de ce qu'on a appelé les "grands défrichements ", c'est-à-dire le mouvement qui, à partir du $x^{\mathrm{e}}$ siècle et sans doute plus tôt, anime l'économie rurale : « essarts », " plaines » évoquent les progrès de la mise en culture par rapport à la pâture, à la friche, à la forêt. Il s'agit de l'élargissement des terroirs les plus anciens, de la création de villages neufs, surtout entre 1100 et 1225 , enfin, du peuplement intercalaire, autour de bergeries, de granges, de bastides, après 1230. Chartes de fondation, chartes de partage ou de peuplement, vies de saints, procès entre propriétaires et utilisateurs, le médiéviste fait feu de tout bois pour éclairer, à travers l'histoire du défrichement, l'histoire de la forêt et de ses usages. Aux sources écrites s'ajoutent, à moins qu'elles ne suppléent à leur absence totale, l'étude des résidus 
floraux et des haies et l'observation du paysage actuel, qui comporte des traces fossilisées des paysages passés dans les terroirs, l'orientation des chemins, etc.

Non moins importants que les défrichements des $\mathrm{X}^{\mathrm{e}}$-XIII ${ }^{\mathrm{e}}$ siècles sont, pour l'histoire des paysages et de la forêt, l'invasion, le retour en force de la végétation sauvage : ainsi, les épisodes correspondant à la grande fracture du temps des Pestes, où les céréales reculent, où les espèces sylvestres progressent, coudriers, bouleaux, puis les hêtres qui reconstituent en certaines régions d'épaisses fûtaies et des « déserts ».

9 Hors des massifs obscurs (Forêt Noire) de l'Europe moyenne, de l'océan à l'Oural, dont la densité n'a pas été substantiellement éclaircie au cours des siècles par leur éloignement des zones de peuplement agricole et urbain, le paysage forestier, au temps des défrichements comme au temps du retour à la friche, est une zone relativement humanisée par les fonctions qu'elle joue dans les équilibres biologiques, et où le pasnage (la pâture des porcs) et, de façon plus large, la présence d'animaux domestiques, contribue à la dégradation: la forêt éclaircie est pénétrée par des prés de fauche, des enclos de pâture, et, dans les parties livrées à la paisson, ne présente pas de belles futaies. On sait avec quelle difficulté l'abbé Suger trouva en forêt d'Yveline en 1130 douze grands arbres capables de fournir des poutres maîtresses pour l'église de Saint-Denis ${ }^{8}$, et voici comment Wolfram von Eschenbach, dans son Parzifal, décrit la chevauchée de Gauvain dans les bois : «Peu à peu, la forêt lui parut toute mélangée ; ici une avancée de bois, là, un champ, mais si étroit qu'on eût pu à peine y dresser une tente ; puis regardant devant lui, il aperçut un pays cultivé ». C'est une zone de transition entre forêt compacte et plaine, plus rarement décrite par les sources littéraires que les forêts profondes où l'on se perd, et où les princes égarés à la chasse rencontrent incognito leurs sujets, le comte d'Anjou, le charbonnier de Loches, ou le seigneur de Milan, le bûcheron de Pavie; où la panique s'empare de l'armée de Charles IV qui remonte d'Italie par le Trentin et se heurte à un inextricable amas d'arbres déracinés et de branches tombées.

10 L'histoire de la forêt est souvent liée, pendant la période médiévale, à la chasse seigneuriale ou royale. C'est une organisation de l'espace sauvage qui remonte au moins à l'époque franque, et, qui, dans une société de pénurie, procure aux puissants le luxe de la viande rôtie, grâce au système des garennes closes (breuils) ${ }^{9}$. A la différence de la silva communis, la grande forêt de chasse est désignée soit par le terme romain de saltus, soit par le terme juridique de "forêt » (forestis), signalé en latin en Ardenne en 648 et qui fait l'objet de débats entre romanistes et germanistes, l'interprétation la plus usuelle du mot, rattaché à forum, insistant sur ce qui est «for » de l'usage commun, et liant des massifs sauvages et stérilisés à une résidence princière, même temporaire. Il est certain que la chasse représente, jusqu'à la Révolution française et au delà, une forme d'activité qui suscite des réactions passionnées, et à propos de laquelle s'affrontent violemment puissants et communautés paysannes. En effet, la chasse noble prime toujours les usages forestiers; la codification de ces usages immémoriaux est le fruit d'un compromis ou d'une victoire après des conflits parfois violents; tout compromis peut être considéré comme une victoire par les villageois, car s'il restreint l'espace ou les modalités des usages, il en affirme aussi le droit imprescriptible.

11 Les paysans prennent du bois pour se chauffer, pour bâtir et clore, pour fabriquer des objets et des outils ${ }^{10}$; les seigneurs tentent de limiter le prélèvement en interdisant par exemple l'utilisation de la cognée d'abattage ou de la scie levée, en comptant par tête le nombre d'échalas coupés pour la vigne, ou le nombre de charretées de bois de chauffage par an. Les paysans usent naturellement de la forêt comme lieu de pacage ; les seigneurs 
admettent l'engraissage des porcs entre octobre et Pâques, mais interdisent les troupeaux de chèvres et exigent que tous les animaux aient regagné la ferme à la fin du jour. On voit par ces détails à quelles tracasseries, à quelle pression sont soumises les communautés d'habitants. Faute de compromis viable entre les exigences des uns et les habitudes des autres, on aboutit souvent au cantonnement ${ }^{11}$, une portion des bois étant remise aux villageois contre l'abandon par eux des usages sur l'ensemble, cette portion donnant naissance aux bois communaux, dont le parcellaire géométrique minuscule tranche aujourd'hui avec la structure d'anciens bois seugneuriaux.

D'autres formes d'exploitation ordinaire du sol et du sous-sol forestier animent le paysage des bois, liées à la présence des métiers villageois ou débouchant sur de véritables exploitations industrielles ${ }^{12}$. La forêt fait vivre les sabotiers et les vanniers, on y collecte les écorces pour le tannage, on y prépare tous les travaux de bois merrain (charpente, menuiserie, charronnerie); on exploite les marnières et sablières, on $y$ fabrique le charbon de bois, on tire du sous-sol la pierre de mine, lorsque les dépôts superficiels affleurent et ne requièrent pas des paysans plus de connaissances techniques que n'en n'avaient leurs ancêtres de la proto-histoire.

13 Aussi laconiques et dispersés que soient les textes relatifs à l'exploitation des mines avant le $\mathrm{xv}^{\mathrm{e}}$ siècle, il est certain que de grands massifs forestiers ont été des lieux d'intense travail métallurgique, et que la couverture végétale a protégé les vestiges et les déchets de l'extraction et de la transformation du métal au cours des siècles. Ce travail s'est déroulé dans le cadre de solidarités régionales, antérieures à la montée en puissance des seigneuries banales : en Champagne, en Normandie, dans la vallée de la Meuse, en Ariège, on aperçoit l'organisation de métiers de férons de grosse forge, c'est-à-dire producteurs de fer, tenant des réunions professionnelles, et réglant des différends entre eux ou avec des tiers sur un territoire qui, dans le cas de la forêt d'Othe en Champagne, correspond à l'extension du massif boisé ${ }^{13}$. A partir de la fin $d u$ XII $^{e}$ siècle, se multiplient les signes d'une sidérurgie seigneuriale en forêt: les abbayes, les comtes, mais aussi de moindres seigneurs créent des forges pour rentabiliser leurs bois.

A l'occasion d'un procès intenté à l'abbé de Clairvaux pour l'exercice de droits qu'il lie à la possession d'une forge dans la forêt de Wassy, on décrit en 1403 l'une de ces entreprises industrielles en plein bois ${ }^{14}$ : " Hostel et forge à faire fer, qui est assise et située esdits bois et forêts de Wassy, et quasi au milieu desdits bois qu'on appelle les usages et abatis avec le droit d'y avoir eyt prendre bois, charbon, terre et mine pour l'usage de leur forge... ». Les lieux sont bornés de fossés et constituent comme une enclave; or les dépendants de Clairvaux ont converti ces lieux forestiers en une clairière de terres labourables afin de faire vivre sur place leurs ouvriers métallurgistes; ils entrent alors en concurrence avec les habitants des proches villages sur les « usages dits les bois batus », et prétendent en outre ne pas payer de redevance aux officiers du roi. Les conflits sont d'autant plus vifs que les sujets de Clairvaux ne raisonnent pas à l'échelle du village, mais dans le cadre d'un espace ouvert sur le marché, et que leur présence contribue à faire monter les prix. On a conservé, à partir du XIV e siècle, des comptabilités d'entreprises métallurgiques forestières éclairantes sur la recherche de débouchés pour le fer produit en forêt: ainsi les grosses forges de la forêt d'Othe en Champagne produisent-elles à la fin du XIV siècle et au début du XVe siècle du fer marchand, qui est vendu à Troyes et jusqu'à $60 \mathrm{~km}$ de là. Comme le fer, le bois et le charbon de bois introduisent par le biais des entrepreneurs laïcs et ecclésiastiques à une économie d'échange à vaste amplitude, qui lie la forêt à la ville. 
15 En prenant l'exemple d'une métropole européenne de 100.000 habitants à la fin du Moyen Age, Venise, port de mer parfaitement relié par la lagune et les fleuves à un vaste bassin d'exploitation forestière, on peut insister sur le fait que c'est la voie d'eau qui fait d'un massif forestier un gisement exploitable ${ }^{15}$. La consommation de bois à Venise dépendait à la fois des conditions climatiques, de la structure urbaine singulière, et des activités de production : outre la demande habituelle d'une grande ville pour la cuisine, la lessive et le bain, le chauffage domestique, on y trouvait réunies toutes les formes de cuisson : fours à pain, à chaux, à briques, savonneries, teintureries, verreries, forges et fonderies, et deux clients privilégiés, la Monnaie et l'Arsenal ; par l'Arsenal, indiquons pour simplifier la construction navale dans l'ensemble de la ville, mais aussi les industries de l'armement pour une capitale et son Empire. Ajoutons que la ville est de bois dans ses structures et ses fondations, ses appontements, ses ponts et ses chenaux. C'est dire que toutes les essences arbustives sont mises à contribution, le merrain pour la tonnellerie d'une grande ville marchande, le chêne, l'orme et le hêtre pour le bâtiment, et, pour la construciton navale, le chêne pour la charpente, le mélèze pour les poutres maîtresses, le sapin pour les mâts, le hêtre pour les rames, etc.

Le ravitaillement de Venise pendant des siècles d'expansion, de conquête et de guerre a mis en oeuvre les ressources en bois d'un arrière pays toujours plus étendu, débordant sur les pays autrichien et slovène; il a nécessité la création d'une administration spécialisée au niveau du territoire, pour réserver des coupes à l'Arsenal, approvisionner les chantiers publics et privés, lutter sans trève pour maintenir un réseau de communication fluviale jusqu'aux lieux de provenance et de flottage du bois.

17 Hors du cas vénitien, qui conjugue toutes les nécessités de la consommation, les forêts les plus denses restaient sans valeur si elles n'étaient pas liées par un système de flottage aux centres marchands : les experts saxons du XVI ${ }^{\mathrm{e}}$ siècle ont calculé que par chariots, le bois de chauffage ne pouvait pas être transporté à plus de quatre heures de route, ni le bois de construction, à plus de sept heures ${ }^{16}$. C'est la construction d'un réseau de voies de flottage qui mit les réserves immenses du Vogtland, à la frontière de Bohême, soit 18.000 hectares de forêt, à la disposition des villes de Saxe et de l'industrie minière et métallurgique du pays; de même pour l'approvisionnement des salines de Halle, on construisit à la fin du $\mathrm{XVI}^{\mathrm{e}}$ siècle un canal latéral à l'Elster sur $90 \mathrm{~km}$, qui fit naître en vingt ans un paysage de désolation : on avait abattu les arbres les plus proches de l'eau, à la hache et non à la scie, laissant sur place sans les déssoucher des moignons de troncs à hauteur d'homme... ${ }^{17}$. Economie de prédation face à des ressources immenses, avec un évident décalage dans l'espace et le temps par rapport à la situation générale de l'Europe occidentale, où le souci d'une gestion prudente des réserves apparaît dès le XIII ${ }^{\mathrm{e}}$ siècle.

Sans doute, la réglementation n'est-t-elle pas le reflet de la réalité ; inversement, les mises en garde alarmistes sur la dévastation des forêts ne doivent pas être prises à la lettre. Mais lorsque le chancelier Poyet conclut vers 1540 à la nécessaire réduction du nombre des forges dans le royaume de France pour éviter un désastre forestier ${ }^{18}$, il faut préciser que sa génération a vécu la généralisation du procédé indirect en sidérurgie, et que des estimations raisonnables imputent à la sidérurgie la consommation de 1/6 des ressources disponibles en futaie du royaume. Face à des exigences contradictoires, la solution, dont on trouve des éléments précurseurs dans les textes publics et privés depuis le XIII $^{\mathrm{e}}$ siècle, fut la mise en coupe réglée, dont la diffusion s'imposa à partir du milieu du $\mathrm{XVI}^{\mathrm{e}}$ siècle : coupe des taillis à dix ans avec réserve des baliveaux, coupe des futaies à cent 
ans. Tout en assurant la relève ${ }^{19}$, on fournissait une offre régulière et contrôlée de bois marchand à tous les niveaux de consommation.

Du soin apporté à l'administration des forêts, on peut apporter un témoignage médiéval exceptionnel, relatif à la ville de Nuremberg ${ }^{20}$. Lorsqu'en 1219, Frédéric II accorde à la ville un grand privilège commercial, il le justifie par le site ingrat : Nuremberg ne possède pas de rivière navigable digne de ce nom, et est enclavée entre deux vastes forêts. Ce qui était conçu comme un handicap devint pour la ville une chance, puisque la présence de minerai de fer à $60 \mathrm{~km}$ à l'Est, dans le haut-Palatinat, se conjuga à l'exploitation des forêts et permit de jeter les bases d'une industrie métallurgique puissante. Mais, vers 1330, la forêt était menacée de disparaître (zergehen); une ordonnance forestière limita strictement l'utilisation du bois, réduisit autoritairement le nombre des charbonniers autorisés à travailler, puis en 1340 , le nombre des forges. La crise énergétique qui résulta de ces restrictions donna naissance à une innovation de grande importance, le semis de forêt ${ }^{21}$. Une personnalité éminente de la ville, Peter Stromer, lui-même maitre de forges et initiateur de l'industrie papetière au Nord des Alpes, avait réussi après de nombreuses expériences à semer des bois de bouleaux, de sapins, de pins et mélèzes. Cette entreprise, destinée à ses propres usines, eut des émules en Saxe, en Bavière et en Autriche, et contribua à répandre les essences de résineux. On a pu démontrer que le paysage forestier actuel du haut-Palatinat est né au moment de la crise d'approvisionnement de la sidérurgie, vers 1380-1400.

L'histoire de la forêt médiévale est encore partielle, et le faisceau de sources dont on dispose pour l'écrire ne permet pas d'espérer la reconstitution d'un paysage continu. On saisit les oscillations de sa substance dans le long terme, liées au rythme d'un monde lâche, qui devint un monde plein, puis se desserre. On perçoit, et mesure parfois, le poids croissant que les besoins énergétiques font peser sur ses ressources, qui - à la différence du pétrole - se reconstituent régulièrement. Paradoxalement, ce sont les industries du feu, les plus prédatrices, comme la métallurgie et la verrerie, qui ont contribué à assurer aux massifs les plus consistants, ceux que la chasse royale avait le mieux protégé des atteintes consommatrices, une gestion raisonnée, qui est le fruit d'un calcul de rentabilité et s'inscrit dans une maîtrise nécessaire de l'espace et du temps.

\section{NOTES}

1. Cf. O. RACKHAM, Ancient Woodland : its History, Vegetation and Uses in England, London, 1981.

2. Par exemple, in "Forges prédatrices, forges protectrices ", Revue géographique des Pyrénées et du Sud-Ouest, 55. 1984, pp. 213-218.

3. Édit de François Ier $(1543 / 18 / V)$ : «Regardant le grand dégât et dépopulation qui se faisait ès bois et forêts de notre royaume pour fournir et entretenir le grand nombre de forges à fer situées près lesdites forêts, nous eussions avisé de faire réduire lesdites forges à nombre certain et suffisant pour fournir de fer notre royaume seulement... ", F. ISAMBERT, Recueil général des anciennes lois françaises depuis l'an 420 jusqu'à la Révolution de 1789, t. XII, p. 810.

4. Une bonne gestion conduit à des progrès sensibles dans la description; que l'on compare les données relatives à la prisée de la forêt de Fontainebleau ou de la forêt d'Orléans dans le premier 
quart du XIV ${ }^{\mathrm{e}}$ siècle et la description de tous les bois du duc de Nevers dans la Réformation de Jean Bodin (nature, étendue, essences, date des dernières coupes, prétention des usages, etc.), G. FOURQUIN, Le domaine royal en Gâtinais d'après la prisée de 1332, Paris, 1963, p. 65 et sq. et P. CORNU, Étude sur les forêts du Nivernais, particulièrement du XV ${ }^{\mathrm{e}}$ à la fin $d u$ XVIII ${ }^{\mathrm{e}}$ siècle, 1906, publié par la Société Académique du Nivernais, 1981, p. 132 et sq.

5. C. HIGOUNET, « Les forêts de l'Europe occidentale du ve siècle à l'an mil ", XIII Settimana di Studi del Centro sull'Alto Medio Evo, Spolète, 1966, pp. 398-399, (carte).

6. O. RACKHAM, «Boschi e storia dei sistemi silvo-pastorali in Inghilterra », Quaderni Storici, 49, 1982, pp. 16-48 ; «Archeology and Land-use History », in D. CORKE (ed.), Epping Forest, Essex Naturalist, n.s., 2, 1978, pp. 58-79.

7. M. LOMBARD, « Le bois dans la Méditerranée musulmane (VII ${ }^{\mathrm{e}}-\mathrm{XI}^{\mathrm{e}}$ siècles) ", Annales E.S.C., 1959, pp. 234-254.

8. SUGER, Libellus de consecratione ecclesiae S. Dyonysii, Migne, t. 186, col. 1244-1245.

9. R. HENNEBICQUE, "Espaces sauvages et chasses royales dans le Nord de la France $\left(\mathrm{VII}^{\mathrm{e}}-\mathrm{IX}^{\mathrm{e}}\right.$ siècles) » in Le paysage rural : réalités et représentations, Actes du $\mathrm{X}^{\mathrm{e}}$ Congrès des historiens médiévistes, Revue du Nord, LXII, 1980, pp. 35-60.

10. M. AUBRUN, « Droits d'usage forestiers et libertés paysannes ( $\mathrm{X}^{\mathrm{e}}-\mathrm{XIII}{ }^{\mathrm{e}}$ siècles). Leur rôle dans la formation de la carte foncière, » Revue Historique, 1988, pp. 377-386.

11. J. RICHARD, «L'origine du cantonnement », Revue des Eaux et Forêts, 1869, p. 400 ; H. RUBNER, "Recherches sur la réorganisation forestière en France (XII ${ }^{\mathrm{e}}$-XIII ${ }^{\mathrm{e}}$ siècles) ", Actes du 88e Congrès national des sociétés savantes (Clermont), 1966, I, pp. 271-279.

12. M. DEVEZE. "L'artisanat et l'industrie en forêt » dans La vie de la forêt française au XVI ${ }^{e}$ siècle, Paris. 1961, I, pp. 129-161.

13. Ph. BRAUNSTEIN, «Les forges champenoises de la comtesse de Flandre (1372-1404) », Annales E.S.C., 1987, pp. 747-777.

14. J.M. ROGER, «Les forges de l'abbaye de Clairvaux à Wassy au Moyen Age ", Actes du $98 e$ Congrès national des sociétés savantes (Saint-Etienne), Mines et métallurgie (XII ${ }^{\mathrm{e}}-\mathrm{XVI}{ }^{\mathrm{e}}$ siècles), I, 1975, pp. 23-59; particulièrement p. 30.

15. Ph BRAUNSTEIN, « De la montagne à Venise : les réseaux du bois au $\mathrm{XV}^{\mathrm{e}}$ siècle ", Mélanges de l'École française de Rome, 100, 1988, 2, pp. 761-799.

16. H. WILSDORF, W. HEUMANN, K. LÖFFLER, «Bergbau-Wald-Flösse : Untersuchungen zur Geschichte der Flösserei ", im Dienste des Montanwesens und zum montanen Transportproblem, Froberger Forschungshefte, 28, Berlin. 1960, p. 36.

17. Ibid., p. 73 et sq.

18. M. FRANCOIS, « Note sur l'industrie métallurgique et les privilèges des maîtres de forge en France au XVI ${ }^{\mathrm{e}}$ siècle ", Mémoires de la Société nationale des Antiquaires de France, IXe série, I, 1944, pp. 113-131. Pour les chiffres de consommation, qui sont à la base du rapport de Poyet, cf. la révision à la baisse de $\mathrm{M}$. DEVEZE, La vie de la forêt française..., II, p. 45. Contemporain de la crise du milieu du XVI ${ }^{\mathrm{e}}$ siècle et proposant les moyens d'y remédier par une bonne gestion des forges, $\mathrm{Cf}$. le rapport, comptes à l'appui, de Louis de Saint-Yon, maître des requêtes de l'hôtel du roi, sur les forges nivernaises de Briffaut et d'Harlot : Les edicts et ordonnances des rois, coustumes des provinces, reglemens, arrests et jugemens notables des eaues et forests, recueillis et divisez en trois livres par le sieur de Sainctyon, Paris. 1610, t. I, pp. 408-412; texte publié, assorti d'un bref commentaire, par G. THUILLIER. in Aspects de l'économie nivernaise au XIXe siècle, Paris, 1961, Annexes, pp. 368-372.

19. Le souci de la relève s'accompagne d'un jugement esthétique sur une forêt bien entretenue, par exemple dans l'ordonnance forestière de l'Empereur Ferdinand, comte de Tirol, pour ses états héréditaires (1551/17/VIII) : « ...Die jungen Baüme, aus denen ein schöner Wald entstehen kann. weder abhacken noch ausreissen... » Schwazer Bergbuch, ed. H. Winckelmann, Wethmar, 1956, p. 49. 
20. G. PFEIFFER, «Wasser und Wald als Faktoren der städtischen Entwicklung ", in Franken, Jahrbuch für fränkische Landesforschung, 32, 1972, pp. 151-170.

21. W. von STROMER, «Die Grosse Hammereinigung vom 7 Januar 1387. Kartell und Innovationen als Antwort auf eine Krise ", in Die Oberpfalz, ein europäisches Eisenzentrum, Bergbauund Industriemuseum Ostbayern Bd. 12/1, 1987, pp. 147-189. Particulièrement p. 173 ; J. LUTZ, « Die ehemaligen Eisenhämmer und Hüttenwerke und die Waldentwicklung » im Nordöstlichen Bayern, Mitteilungen aus Forstwirtschaft und Forstwissenschaft, 12, 1941, pp. 277-294 ; R. J. GLEITZMANN, « Der Einfluss der Montanwirtschaft auf die Waldentwicklung Mitteleuropas. Stand und Aufgaben der Forschung ", Montanwirtschaft Mitteleuropas vom 12. bis 17. Jahrhundert, Forschungsproblem, ed. E. WESTERMANN, W. KROKER, Der Anschnitt, Beiheft 2, Bochum. 1984, pp. 24-39.

\section{AUTEUR}

\section{PHILIPPE BRAUNSTEIN}

Philippe BRAUNSTEIN est directeur d'études au Centre de Recherches Historiques. 